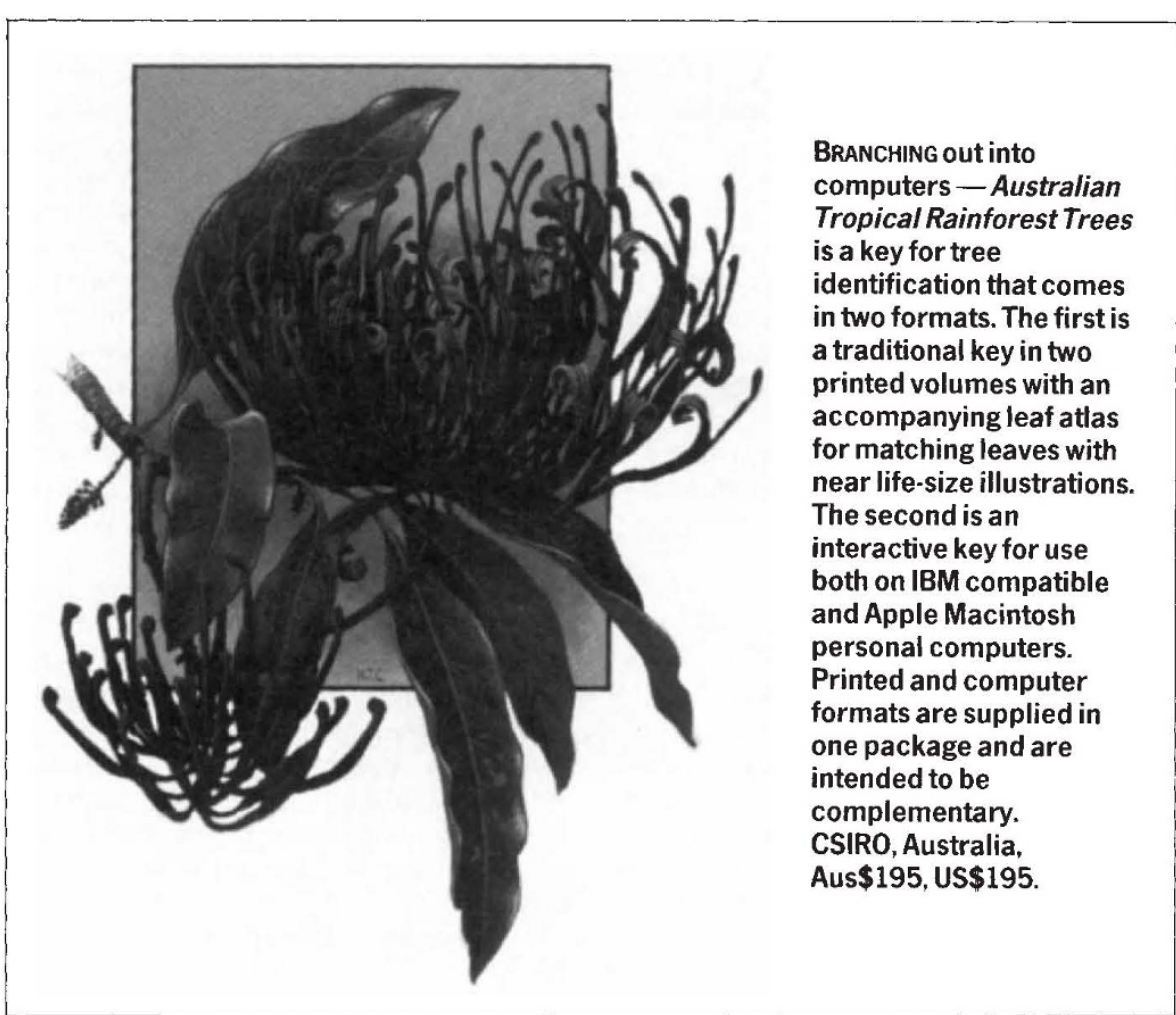

\section{Fringe physics}

Peter Holland

The Undivided Universe: An Ontological Interpretation of Quantum Theory. By D. Bohm and B. J. Hiley. Routledge: 1993. Pp. 397. £25, \$29.95.

WITH the death of David Bohm in 1992 , twentieth-century theoretical physics lost one of its most important thinkers. In conventional physics Bohm is known for his contributions to plasma physics, his classic text on quantum theory and as codiscoverer of the Aharonov-Bohm effect. But his most enduring bestowal may well prove to be his work in an area many have felt to be on the fringes of acceptable physics: the interpretation of quantum mechanics that he first proposed in 1952 following the earlier work of Louis de Broglie. After years of neglect, the theory is undergoing a renaissance and this book presents what Hiley states in the preface to be Bohm's final thoughts on it.

Bohm's idea is that the electron is a point particle as in classical mechanics, but that its trajectory is influenced by what he called the quantum potential, a new type of non-classical physical field derived from the wave function. He thus provides an 'ontology', a model of how the world is built, that contrasts with the conventional interpretation which is concerned solely with our (statistical) knowledge of the world. In his original papers he showed how the statistical predictions of quantum mechanics could be generated through an ensemble of individually well defined causal motions.

The book is intended to bring this idea up to date and describes in mathematical terms how to develop ontological explanations for many classic quantum phenomena, including transitions, interference and relativistic effects. A careful analysis is given to show that the causal theory cannot be excluded on grounds of naturalness or economy of concepts, for all the interpretations of quantum mechanics proposed so far entail assumptions that go beyond the basic formalism. But Bohm's theory has the major advantage of conceptual clarity, and the paradoxes that afflict other views simply do not arise.

One reason the theory has been dismissed is that it implies a deep interconnectedness of distant systems, or 'nonlocality', although, as pointed out by the authors, the orthodox view also involves nonlocality in an essential way. Indeed, the authors show a deeper appreciation of the meaning of the conventional interpretation than do many of its proponents. Most contemporary presentations still imply that history bequeathed us a homogeneous quantum world view. It is rarely remarked that Bohr, Heisenberg and von Neumann differed significantly in their analyses, von Neumann's concept of the 'quantum state' of an atomic system being incompatible with Bohr's notion that systems cannot be conceived independently of the means by which they are obserrved. It would help students considerably if this confusion in the official quantum story was openly admitted.

The authors attempt to find a metaphor that adequately encapsulates what they feel is the novelty of quantum theory as revealed in this model. Their solution is to regard the quantum field as a repository of 3 potentially active information, the electron being likened to a ship moving under its own energy and guided by a radar wave of considerably less energy. It is a view that some may find unpalatable because it seems to read more into the mathematics than is actually there. And there is a curious empiricist tone to some of the statements, such as the assertion that the only intrinsic property of an electron is its position because only this can be measured without disturbance. These ideas are interesting but there are other points of view consistent with the basic model that are not represented and the book does not provide a comprehensive review of the field as it now exists. This subject is beginning to flourish and the inclusion of other relevant recent work would have improved the presentation. There also seem to be some oversights in properly attributing previous works to their orginal sources.

The causal theory provides a simple criterion for judging when quantum systems will display classical behaviour (be governed by Newton's laws) through the relative effectiveness of the quantum potential. This suggests the view expressed in the title of the book that the Universe is an 'undivided whole', a system that is basically quantum mechanical yet capable of the classical behaviour we encounter at the scale of macroexperience. It is a compelling picture, but is it true that all the phenomena met at the macro-scale that are correctly described by classical physics are special cases in an enveloping quantum description? Although not mentioned in the book, it is a bonus of the causal model that it intimates a more subtle connection between the two domains, that classical phenomena cannot always be obtained in some limit, and it is unclear whether the Universe really is 'undivided' in the sense that the author intends.

Should we be alarmed that both causal and non-causal theories can account for the same empirical quantum data? Some may fear the lack of certainty in science, but it is closed minds that are harmful, not the proliferation of ideas. It was contemplation of Bohm's theory 30 years ago that prompted Bell to formulate his famous theorem distinguishing local from nonlocal theories. I hope that this book will further stimulate current investigations into causal theories in quantum physics.

Peter Holland is in the Laboratoire de Gravitation et Cosmologie Relativistes, Université Pierre et Marie Curie, Boîte 142, 4 Place Jussieu, 75252 Paris Cedex 05. France. 